\title{
Emden-Fowler-type neutral differential equations: oscillatory properties of solutions
}

\section{Omar Bazighifan ${ }^{1,2^{*}}$ (]) and Alanoud Almutairi ${ }^{3}$}

\section{"Correspondence:}

o.bazighifan@gmail.com

'Department of Mathematics,

Faculty of Science, Hadhramout

University, Hadhramout 50512, Yemen

2Department of Mathematics, Faculty of Education, Seiyun University, Hadhramout 50512, Yemen

Full list of author information is available at the end of the article

\begin{abstract}
In this paper, we study the oscillation of a class of fourth-order Emden-Fowler delay differential equations with neutral term. Using the Riccati transformation and comparison method, we establish several new oscillation conditions. These new conditions complement a number of results in the literature. We give examples to illustrate our main results.
\end{abstract}

MSC: 34C10; 34K11

Keywords: Neutral differential equations; Oscillation; Fourth-order equations

\section{Introduction}

In this paper, we study the oscillatory properties of solutions of the following fourth-order neutral differential equation:

$$
X_{t}^{\prime}+q(t) x^{\left(p_{2}-1\right)}(\sigma(t))=0, \quad t \geq t_{0}
$$

where $X_{t}=a(t)\left(y^{\prime \prime \prime}(t)\right)^{\left(p_{1}-1\right)}$ and $y(t):=x(t)+r(t) x(\delta(t))$. We make the following assumptions:

$\mathrm{N} 1: r \in C\left[t_{0}, \infty\right), 0 \leq r(t)<r_{0}<\infty$,

$\mathrm{N} 2: \delta, \sigma, q \in C\left[t_{0}, \infty\right), q(t)>0, \delta(t) \leq t, \lim _{t \rightarrow \infty} \delta(t)=\lim _{t \rightarrow \infty} \sigma(t)=\infty$,

N3: $a \in C\left[t_{0}, \infty\right), a(t)>0, a^{\prime}(t) \geq 0$, and

$$
\int_{t_{0}}^{\infty} \frac{1}{a^{1 /\left(p_{1}-1\right)}(s)} \mathrm{d} s=\infty
$$

N4: $p_{i}>1, i=1,2$, are constants, and

$$
p_{1}:= \begin{cases}2 & \text { if } p_{2} \leq 2 \\ 1+2^{\beta-1} & \text { if } p_{2}>2\end{cases}
$$

By a solution of (1) we mean a function $x \in C^{3}[t, \infty), t \geq t_{0}$, that has the property $a(t)\left(y^{\prime \prime \prime}(t)\right)^{\alpha} \in C^{1}\left[t_{0}, \infty\right)$ and satisfies (1) on $\left[t_{0}, \infty\right)$.

(c) The Author(s) 2021. This article is licensed under a Creative Commons Attribution 4.0 International License, which permits use, sharing, adaptation, distribution and reproduction in any medium or format, as long as you give appropriate credit to the original author(s) and the source, provide a link to the Creative Commons licence, and indicate if changes were made. The images or other third party material in this article are included in the article's Creative Commons licence, unless indicated otherwise in a credit line to the material. If material is not included in the article's Creative Commons licence and your intended use is not permitted by statutory regulation or exceeds the permitted use, you will need to obtain permission directly from the copyright holder. To view a copy of this licence, visit http://creativecommons.org/licenses/by/4.0/. 
The study of differential equations has been the object of many researchers over the last decades. Different approaches and various techniques are adopted to investigate the qualitative properties of their solutions. Recently, driven by their widespread applications, the investigation of fourth-order differential equations has drawn significant attention. The existence uniqueness, stability, and oscillation of solutions were the main features that attracted consideration [1-3].

In spite of the increasing interest in the study of second-order differential equations, the oscillation and nonoscillation of solutions for differential equations are still considered as an open area to investigate [4-9]. Equations with neutral terms are of particular significance as they arise in many applications including systems of control, electrodynamics, mixing liquids, neutron transportation, networks, and population models. In the qualitative analysis of such systems, indeed, the oscillatory behavior of solutions of equations, where the rate of the growth depends not only on the current and the past states but also on the rate of change in the past, play an important role [10-14]. In the light of this motivation and justification, different results have been reported regarding the asymptotic behavior of higher-order differential equations with neutral terms [15-18]. For relevant results on the application of oscillation theory, the reader can consult [19-21]. In the past 20 years, there have been a lot of research results on the oscillation of differential equations. As a matter of fact, Eq. (1) is a natural of the half-linear/Emden-Fowler differential equation (including the related differential equation), which arises in a variety of realworld problems such as the study of $p$-Laplace equations, non-Newtonian fluid theory, the turbulent flow of a polytrophic gas in a porous medium, and so on; see, for example, the papers $[22,23]$ for more detail.

The authors in $[24,25]$ considered the equation

$$
y^{(r)}(t)+q(t) x(\sigma(t))=0
$$

and proved that it is oscillatory if

$$
\liminf _{t \rightarrow \infty} \int_{\sigma(t)}^{t} K(s) \mathrm{d} s>2^{(r-1)(r-2)} \frac{(r-1)}{\mathrm{e}}
$$

and

$$
\liminf _{t \rightarrow \infty} \int_{\sigma(t)}^{t} K(s) \mathrm{d} s>\frac{(r-1) !}{\mathrm{e}}
$$

where $K(t):=\sigma^{r-1}(t)(1-b(\sigma(t))) q(t)$, and $r$ is an even.

In $[26,27]$ the authors proved that the equation

$$
\left(a(t)\left(y^{(n-1)}(t)\right)^{\alpha}\right)^{\prime}+q(t) x^{\alpha}(\sigma(t))=0
$$

is oscillatory if

$$
\liminf _{t \rightarrow \infty} \int_{\delta^{-1}(\sigma(t))}^{t} \frac{q(s)}{a(s)}\left(s^{n-1}\right)^{\alpha} \mathrm{d} s>\left(\frac{1}{\sigma_{0}}+\frac{p_{0}^{\alpha}}{\sigma_{0} \delta_{0}}\right) \frac{((n-1) !)^{\alpha}}{\mathrm{e}}
$$


and

$$
\liminf _{t \rightarrow \infty} \int_{\delta^{-1}(\eta(t))}^{t}\left(\frac{\left(\tau^{-1}(\eta(s))\right)^{n-1}}{a^{1 / \alpha}\left(\delta^{-1}(\eta(s))\right)}\right)^{\alpha} q(s) P_{n}^{\alpha}(\sigma(s)) \mathrm{d} s>\frac{((n-1) !)^{\alpha}}{\mathrm{e}},
$$

where $\eta \in C^{1}\left(\left[t_{0}, \infty\right), \mathbb{R}\right)$ and $\widehat{q}(t):=\min \left\{q\left(\sigma^{-1}(t)\right), q\left(\sigma^{-1}(\delta(t))\right)\right\}$.

Li et al. [28] studied the oscillatory and asymptotic behavior of higher-order EmdenFowler neutral differential equations using the Riccati substitution together with integral averaging technique. Bazighifan [29] established sufficient conditions for the oscillation of all solutions of (1) and used the comparison method with second-order equations. Agarwal et al. [30] gave some results providing information on the asymptotic behavior of solutions of fourth-order Emden-Fowler neutral differential equations. This time the authors used the comparison method with first- and second-order equations. In [31] the authors considered the equation

$$
\left(a(t) X\left(y^{(n-1)}(t)\right)\right)^{\prime}+q(t) F(y(\sigma(t)))=0,
$$

where $F=|s|^{p-2} s$, and obtained some new oscillation conditions.

In particular, there has been interest from many researchers to study the oscillatory behavior of this type of equation; see [32-39].

In this paper, we establish oscillatory properties of solutions of (1) and give some examples for applying the criteria.

\section{Preliminaries}

We first provide some notations which help us to easily display the results. Moreover, we present some auxiliary lemmas.

Lemma 2.1 ([40]) If $u^{(i)}(t)>0, i=0,1, \ldots, j$, and $u^{(j+1)}(t)<0$ eventually, then, for every $\varepsilon_{1} \in(0,1), u(t) / u^{\prime}(t) \geq \varepsilon_{1} t / j$ eventually.

Lemma 2.2 ([41] $)$ Let $u \in C^{j}\left(\left[t_{0}, \infty\right),(0, \infty)\right)$. Assume that $u^{(j)}(t)$ is of fixed sign and not identically zero on $\left[t_{0}, \infty\right)$ and that there exists $t_{1} \geq t_{0}$ such that $u^{(j-1)}(t) u^{(j)}(t) \leq 0$ for all $t \geq t_{1}$. If $\lim _{t \rightarrow \infty} u(t) \neq 0$, then for every $\mu \in(0,1)$, there exists $t_{\mu} \geq t_{1}$ such that

$$
u(t) \geq \frac{\mu}{(j-1) !} t^{j-1}\left|u^{(j-1)}(t)\right| \quad \text { for } t \geq t_{\mu} .
$$

Lemma 2.3 ([42, Lemmas 1 and 2]) Let $m_{1}, m_{2} \geq 0$. Then

$$
\left(m_{1}+m_{2}\right)^{\beta} \leq \begin{cases}2^{\beta-1}\left(m_{1}^{\beta}+m_{2}^{\beta}\right) & \text { for } \beta \geq 1, \\ m_{1}^{\beta}+m_{2}^{\beta} & \text { for } \beta \leq 1 .\end{cases}
$$

For convenience, we impose the following hypothesis:

(H1) $x$ is an eventually positive solution of (1).

\section{Main results}

Theorem 3.1 Assume that

$$
\left(\sigma^{-1}(t)\right)^{\prime} \geq \sigma_{0}>0 \quad \text { and } \quad \delta^{\prime}(t) \geq \delta_{0}>0 .
$$


If

$$
\begin{aligned}
\eta^{\prime}(t) & +\frac{1}{\left(p_{1}-1\right)}\left(\frac{\mu t^{3}}{6 a^{1 /\left(p_{1}-1\right)}(t)}\right)^{\left(p_{2}-1\right)}\left(\frac{\sigma_{0} \delta_{0}}{\delta_{0}+r_{0}^{\left(p_{2}-1\right)}}\right)^{\left(p_{2}-1\right) /\left(p_{1}-1\right)} \\
\times & \widehat{q}(t) \eta^{\left(p_{2}-1\right) /\left(p_{1}-1\right)}\left(\delta^{-1}(\sigma(t))\right) \leq 0
\end{aligned}
$$

is oscillatory, where

$$
\widehat{q}(t):=\min \left\{q\left(\sigma^{-1}(t)\right), q\left(\sigma^{-1}(\delta(t))\right)\right\}
$$

then (1) is oscillatory.

Proof Let $t$ be a nonoscillatory solution of (1) on $\left[t_{0}, \infty\right)$. Then $t>0$, and there exists $t_{1} \geq t_{0}$ such that $x(t)>0, x(\delta(t))>0$, and $x(\sigma(t))>0$ for $t \geq t_{1}$. Since $a^{\prime}(t)>0$, we have

$$
\begin{aligned}
& y(t)>0, \quad y^{\prime}(t)>0, \quad y^{\prime \prime \prime}(t)>0, \quad y^{(4)}(t)<0, \quad \text { and } \\
& \left(a(t)\left(y^{\prime \prime \prime}(t)\right)^{\left(p_{1}-1\right)}\right)^{\prime} \leq 0
\end{aligned}
$$

for $t \geq t_{1}$. From (1) we get

$$
\frac{1}{\left(\sigma^{-1}(t)\right)^{\prime}}\left(a\left(\sigma^{-1}(t)\right)\left(y^{\prime \prime \prime}\left(\sigma^{-1}(t)\right)\right)^{\left(p_{1}-1\right)}\right)^{\prime}+q\left(\sigma^{-1}(t)\right) x^{\left(p_{2}-1\right)}(t)=0 .
$$

By Lemma 2.3 and the definition of $y$ we obtain

$$
\begin{aligned}
y^{\left(p_{2}-1\right)}(t) & =(x(t)+r(t) x(\delta(t)))^{\left(p_{2}-1\right)} \\
& \leq\left(p_{1}-1\right)\left(x^{\left(p_{2}-1\right)}(t)+r_{0}^{\left(p_{2}-1\right)} x^{\left(p_{2}-1\right)}(\delta(t))\right) .
\end{aligned}
$$

From (10) and (11) we obtain

$$
\begin{aligned}
0= & \frac{1}{\left(\sigma^{-1}(t)\right)^{\prime}}\left(a\left(\sigma^{-1}(t)\right)\left(y^{\prime \prime \prime}\left(\sigma_{j}^{-1}(t)\right)\right)^{\left(p_{1}-1\right)}\right)^{\prime}+q\left(\sigma^{-1}(t)\right) x^{\left(p_{2}-1\right)}(t) \\
& +r_{0}^{\left(p_{2}-1\right)}\left(\frac{1}{\left(\sigma^{-1}(\delta(t))\right)^{\prime}}\left(a\left(\sigma^{-1}(\delta(t))\right)\left(y^{\prime \prime \prime}\left(\sigma^{-1}(\delta(t))\right)\right)^{\left(p_{1}-1\right)}\right)^{\prime}\right. \\
& \left.+q\left(\sigma^{-1}(\delta(t))\right) x^{\left(p_{2}-1\right)}(\delta(t))\right) \\
= & \frac{\left(a\left(\sigma^{-1}(t)\right)\left(y^{\prime \prime \prime}\left(\sigma^{-1}(t)\right)\right)^{\left.\left(p_{1}-1\right)\right)^{\prime}}\right.}{\left(\sigma^{-1}(t)\right)^{\prime}}+r_{0}^{\left(p_{2}-1\right)} \frac{\left(a\left(\sigma^{-1}(\delta(t))\right)\left(y^{\prime \prime \prime}\left(\sigma^{-1}(\delta(t))\right)\right)^{\left(p_{1}-1\right)}\right)^{\prime}}{\left(\sigma^{-1}(\delta(t))\right)^{\prime}} \\
& +q\left(\sigma^{-1}(t)\right) x^{\left(p_{2}-1\right)}(t)+r_{0}^{\left(p_{2}-1\right)} q\left(\sigma^{-1}(\delta(t))\right) x^{\left(p_{2}-1\right)}(\delta(t)) \\
\geq & \frac{\left(a\left(\sigma^{-1}(t)\right)\left(y^{\prime \prime \prime}\left(\sigma^{-1}(t)\right)\right)^{\left(p_{1}-1\right)}\right)^{\prime}}{\left(\sigma^{-1}(t)\right)^{\prime}}+r_{0}^{\left(p_{2}-1\right)} \frac{\left(a\left(\sigma^{-1}(\delta(t))\right)\left(y^{\prime \prime \prime}\left(\sigma^{-1}(\delta(t))\right)\right)^{\left(p_{1}-1\right)}\right)^{\prime}}{\left(\sigma^{-1}(\delta(t))\right)^{\prime}} \\
& +\frac{1}{\left(p_{1}-1\right)} \widehat{q}(t) y^{\left(p_{2}-1\right)}(t),
\end{aligned}
$$


which, together with (7), gives

$$
\begin{aligned}
& \frac{1}{\sigma_{0}}\left(a\left(\sigma^{-1}(t)\right)\left(y^{\prime \prime \prime}\left(\sigma_{j}^{-1}(t)\right)\right)^{\left(p_{1}-1\right)}\right)^{\prime} \\
& \quad+\frac{r_{0}^{\left(p_{2}-1\right)}}{\sigma_{0} \delta_{0}}\left(a\left(\sigma^{-1}(\delta(t))\right)\left(y^{\prime \prime \prime}\left(\sigma^{-1}(\delta(t))\right)\right)^{\left(p_{1}-1\right)}\right)^{\prime}+\frac{1}{\left(p_{1}-1\right)} \widehat{q}(t) y^{\left(p_{2}-1\right)}(t) \leq 0 .
\end{aligned}
$$

Since $y^{\prime}(t)>0$, we find $\lim _{t \rightarrow \infty} y(t) \neq 0$, and by Lemma 2.2 we obtain

$$
y(t) \geq \frac{\mu}{6} t^{3} y^{\prime \prime \prime}(t)
$$

Combining (12) and (13), we see that

$$
\begin{aligned}
& \frac{1}{\sigma_{0}}\left(a\left(\sigma^{-1}(t)\right)\left(y^{\prime \prime \prime}\left(\sigma_{j}^{-1}(t)\right)\right)^{\left(p_{1}-1\right)}\right)^{\prime}+\frac{r_{0}^{\left(p_{2}-1\right)}}{\sigma_{0} \delta_{0}}\left(a\left(\sigma^{-1}(\delta(t))\right)\left(y^{\prime \prime \prime}\left(\sigma^{-1}(\delta(t))\right)\right)^{\left(p_{1}-1\right)}\right)^{\prime} \\
& +\frac{1}{\left(p_{1}-1\right)} \widehat{q}(t)\left(\frac{\mu}{6} t^{3}\right)^{\left(p_{2}-1\right)}\left(y^{\prime \prime \prime}(t)\right)^{\left(p_{2}-1\right)} \leq 0 .
\end{aligned}
$$

Setting

$$
\eta(t):=\frac{1}{\sigma_{0}} a\left(\sigma^{-1}(t)\right)\left(y^{\prime \prime \prime}\left(\sigma_{j}^{-1}(t)\right)\right)^{\left(p_{1}-1\right)}+\frac{r_{0}^{\left(p_{2}-1\right)}}{\sigma_{0} \delta_{0}} a\left(\sigma^{-1}(\delta(t))\right)\left(y^{\prime \prime \prime}\left(\sigma^{-1}(\delta(t))\right)\right)^{\left(p_{1}-1\right)},
$$

we easily see that

$$
\eta\left(\delta^{-1}(\sigma(t))\right) \leq\left(\frac{\delta_{0}+r_{0}^{\left(p_{2}-1\right)}}{\sigma_{0} \delta_{0}}\right) a(t)\left(y^{\prime \prime \prime}(t)\right)^{\left(p_{1}-1\right)} .
$$

From (14) we find

$$
\begin{aligned}
\eta^{\prime}(t) & +\frac{1}{\left(p_{1}-1\right)}\left(\frac{\mu t^{3}}{6 a^{1 /\left(p_{1}-1\right)}(t)}\right)^{\left(p_{2}-1\right)}\left(\frac{\sigma_{0} \delta_{0}}{\delta_{0}+r_{0}^{\left(p_{2}-1\right)}}\right)^{\left(p_{2}-1\right) /\left(p_{1}-1\right)} \\
& \times \widehat{q}(t) \eta^{\left(p_{2}-1\right) /\left(p_{1}-1\right)}\left(\delta^{-1}(\sigma(t))\right) \leq 0,
\end{aligned}
$$

which is a contradiction.

Theorem 3.2 Assume that (7) holds. If

$$
\vartheta^{\prime}(t)+\frac{1}{\left(p_{1}-1\right)}\left(\frac{\mu t^{3}}{6 a^{1 /\left(p_{1}-1\right)}(t)}\right)^{\left(p_{2}-1\right)}\left(\frac{\sigma_{0} \delta_{0}}{\delta_{0}+r_{0}^{\left(p_{2}-1\right)}}\right) \widehat{q}(t) \vartheta^{\left(p_{2}-1\right) /\left(p_{1}-1\right)}(\sigma(t)) \leq 0
$$

is oscillatory, then (1) is oscillatory.

Proof It is known that (14) holds in the proof of Theorem 3.1. If we set

$$
\vartheta(t):=a\left(\sigma^{-1}(t)\right)\left(y^{\prime \prime \prime}\left(\sigma^{-1}(t)\right)\right)^{\left(p_{1}-1\right)},
$$

then $\vartheta$ is a positive solution of (15), which is a contradiction. 
Corollary 3.1 Let $p_{1}=p_{2}$, and let (7) hold. If $a(t) \leq t$ and

$$
\liminf _{t \rightarrow \infty} \int_{a(t)}^{t} \frac{s^{3\left(p_{1}-1\right)}}{a(s)} \widehat{q}(s) \mathrm{d} s>\left(\frac{\delta_{0}+r_{0}^{\left(p_{1}-1\right)}}{\sigma_{0} \delta_{0}}\right) \frac{\left(p_{1}-1\right) 6^{\left(p_{1}-1\right)}}{\mathrm{e}}
$$

where $a(t)=\delta^{-1}(\sigma(t))$ or $\sigma(t)$, then (1) is oscillatory.

Theorem 3.3 Let $r_{0}<1$ and $\sigma(t) \leq t$. Iffor some $\mu \in(0,1)$,

$$
\psi^{\prime}(t)+\left(1-r_{0}\right)^{\left(p_{2}-1\right)}\left(\frac{\mu \sigma^{3}(t)}{6 a^{1 /\left(p_{1}-1\right)}(\sigma(t))}\right)^{\left(p_{2}-1\right)} q(t) \psi^{\left(p_{2}-1\right) /\left(p_{1}-1\right)}(\sigma(t))=0
$$

is oscillatory, then (1) is oscillatory.

Proof It is known that (9) holds in the proof of Theorem 3.1. By the definition of $y$ we find

$$
\begin{aligned}
x(t) & \geq y(t)-r_{0} x(\delta(t)) \geq y(t)-r_{0} y(\delta(t)) \\
& \geq\left(1-r_{0}\right) y(t),
\end{aligned}
$$

which, together with (1), gives

$$
\left(a(t)\left(y^{\prime \prime \prime}(t)\right)^{\left(p_{1}-1\right)}\right)^{\prime}+q(t)\left(1-r_{0}\right)^{\left(p_{2}-1\right)} y^{\left(p_{2}-1\right)}(\sigma(t)) \leq 0
$$

From Lemma 2.2 we obtain

$$
y(t) \geq \frac{\mu}{6} t^{3} y^{\prime \prime \prime}(t)
$$

Combining (18) and (19), we get

$$
\left(a(t)\left(y^{\prime \prime \prime}(t)\right)^{\left(p_{1}-1\right)}\right)^{\prime}+q(t)\left(1-r_{0}\right)^{\left(p_{2}-1\right)}\left(\frac{\mu}{6} \sigma^{3}(t)\right)^{\left(p_{2}-1\right)}\left(y^{\prime \prime \prime}(\sigma(t))\right)^{\left(p_{2}-1\right)} \leq 0 .
$$

If we set $\psi:=a\left(y^{\prime \prime \prime}\right)^{\left(p_{1}-1\right)}$, then

$$
\psi^{\prime}(t)+\left(1-r_{0}\right)^{\left(p_{2}-1\right)}\left(\frac{\mu \sigma^{3}(t)}{6 a^{1 /\left(p_{1}-1\right)}(\sigma(t))}\right)^{\left(p_{2}-1\right)} q(t) \psi^{\left(p_{2}-1\right) /\left(p_{1}-1\right)}(\sigma(t)) \leq 0 .
$$

In view of [37, Corollary 1], equation (17) also has a positive solution, which is a contradiction.

Corollary 3.2 Let $p_{1}=p_{2}, r_{0}<1$, and $\sigma(t) \leq t$. If

$$
\liminf _{t \rightarrow \infty} \int_{\sigma_{j}(t)}^{t} \frac{\sigma^{3\left(p_{1}-1\right)}(s)}{a(\sigma(s))} q(s) \mathrm{d} s>\frac{6^{\left(p_{1}-1\right)}}{\left(1-r_{0}\right)^{\left(p_{1}-1\right)} \mathrm{e}}
$$

then (1) is oscillatory. 
Lemma 3.1 If $(H 1)$ holds, then

$$
\begin{aligned}
\phi_{1}^{\prime}(t) \leq & \frac{\varpi_{1}^{\prime}(t)}{\varpi_{1}(t)} \phi_{1}(t)-\varpi_{1}(t) q(t)\left(1-r_{0}\right)^{\left(p_{2}-1\right)} y^{p_{2}-p_{2}}(t) \varepsilon_{1}\left(\frac{\sigma_{j}(t)}{t}\right)^{3\left(p_{2}-1\right)} \\
& -\left(p_{1}-1\right) \mu_{1} \frac{t^{2}}{2 a^{1 /\left(p_{1}-1\right)}(t) \varpi_{1}^{1 /\left(p_{1}-1\right)}(t)} \phi_{1}^{\frac{p_{1}}{\left(p_{1}-1\right)}}(t)
\end{aligned}
$$

for some $\mu_{1}, \varepsilon_{1} \in(0,1)$ and every $M_{1}>0$, where

$$
\Psi(t):=M_{1}^{p_{2}-p_{1}} \varpi_{1}(t) q(t)\left(1-r_{0}\right)^{\left(p_{2}-1\right)}\left(\frac{\sigma(t)}{t}\right)^{3\left(p_{2}-1\right)} .
$$

Proof Let $(H 1)$ hold. In the case where $y^{\prime \prime}(t)>0$, let

$$
\phi_{1}(t):=\varpi_{1}(t) \frac{a(t)\left(y^{\prime \prime \prime}(t)\right)^{\left(p_{1}-1\right)}}{y^{\left(p_{1}-1\right)}(t)}>0 .
$$

From (18) we find

$$
\begin{aligned}
\phi_{1}^{\prime}(t) \leq & \frac{\varpi_{1}^{\prime}(t)}{\varpi_{1}(t)} \phi_{1}(t)-\varpi_{1}(t) q(t)\left(1-r_{0}\right)^{\left(p_{2}-1\right)} \frac{y^{\left(p_{2}-1\right)}(\sigma(t))}{y^{\left(p_{1}-1\right)}(t)} \\
& -\left(p_{1}-1\right) \varpi_{1}(t) \frac{a(t)\left(y^{\prime \prime \prime}(t)\right)^{\left(p_{1}-1\right)}}{y^{p_{1}}(t)} y^{\prime}(t) .
\end{aligned}
$$

Using Lemma 2.1, we obtain $y(t) \geq \frac{t}{3} y^{\prime}(t)$, and hence

$$
\frac{y\left(\sigma_{j}(t)\right)}{y(t)} \geq \varepsilon_{1} \frac{\sigma^{3}(t)}{t^{3}}
$$

Using Lemma 2.2, we get

$$
y^{\prime}(t) \geq \frac{\mu_{1}}{2} t^{2} y^{\prime \prime \prime}(t)
$$

for all $\mu_{1} \in(0,1)$. Thus by (22), (23), and (24) we obtain

$$
\begin{aligned}
\phi_{1}^{\prime}(t) \leq & \frac{\varpi_{1}^{\prime}(t)}{\varpi_{1}(t)} \phi_{1}(t)-\varpi_{1}(t) q(t)\left(1-r_{0}\right)^{\left(p_{2}-1\right)} y^{p_{2}-p_{2}}(t) \varepsilon_{1}\left(\frac{\sigma_{j}(t)}{t}\right)^{3\left(p_{2}-1\right)} \\
& -\left(p_{1}-1\right) \mu_{1} \frac{t^{2}}{2 a^{1 /\left(p_{1}-1\right)}(t) \varpi_{1}^{1 /\left(p_{1}-1\right)}(t)} \phi_{1}^{\frac{p_{1}}{\left(p_{1}-1\right)}}(t) .
\end{aligned}
$$

This completes the proof.

Lemma 3.2 If $(H 1)$ holds, then

$$
\phi_{2}^{\prime}(t) \leq-\Psi_{1}(t)+\frac{\varpi^{\prime}(t)}{\varpi(t)} \vartheta(t)-\frac{1}{\varpi(t)} \phi_{2}^{2}(t)
$$


for some $\varepsilon_{1} \in(0,1)$ and every $M_{2}>0$, where

$$
\begin{aligned}
\Psi_{1}(t):= & \left(\left(1-r_{0}\right) \varepsilon_{1}\right)^{\left(p_{2}-1\right) /\left(p_{1}-1\right)} \varpi(t) M_{2}^{\left(p_{2}-p_{1}\right) /\left(p_{1}-1\right)} \\
& \times \int_{t}^{\infty}\left(\frac{1}{a(t)} \int_{t}^{\infty} q(s) \frac{\sigma^{\left(p_{2}-1\right)}(s)}{s^{\left(p_{2}-1\right)}} \mathrm{d} s\right)^{1 /\left(p_{1}-1\right)} \mathrm{d} t
\end{aligned}
$$

Proof Let $(H 1)$ hold. In the case where $y^{\prime \prime}(t)<0$, integrating (18) from $t$ to $t$, we find

$$
a(t)\left(y^{\prime \prime \prime}(t)\right)^{\left(p_{1}-1\right)}-a(t)\left(y^{\prime \prime \prime}(t)\right)^{\left(p_{1}-1\right)} \leq-\int_{t}^{t} q(s)\left(1-r_{0}\right)^{\left(p_{2}-1\right)} y^{\left(p_{2}-1\right)}(\sigma(s)) \mathrm{d} s .
$$

By Lemma 2.1 we get $y(t) \geq x y^{\prime}(t)$, and hence

$$
y(\sigma(t)) \geq \varepsilon_{1} \frac{\sigma(t)}{t} y(t)
$$

For (26), letting $t \rightarrow \infty$ and using (27), we get

$$
a(t)\left(y^{\prime \prime \prime}(t)\right)^{\left(p_{1}-1\right)} \geq\left(\left(1-r_{0}\right) \varepsilon_{1}\right)^{\left(p_{2}-1\right)} y^{\left(p_{2}-1\right)}(t) \int_{t}^{\infty} q(s) \frac{\sigma_{j}^{\left(p_{2}-1\right)}(s)}{s^{\left(p_{2}-1\right)}} \mathrm{d} s .
$$

Integrating (28) from $t$ to $\infty$, we get

$$
\begin{aligned}
y^{\prime \prime}(t) \leq & -\left(\left(1-r_{0}\right) \varepsilon_{1}\right)^{\left(p_{2}-1\right) /\left(p_{1}-1\right)} y^{\left(p_{2}-1\right) /\left(p_{1}-1\right)}(t) \\
& \times \int_{t}^{\infty}\left(\frac{1}{a(t)} \int_{t}^{\infty} q(s) \frac{\sigma^{\left(p_{2}-1\right)}(s)}{s^{\left(p_{2}-1\right)}} \mathrm{d} s\right)^{1 /\left(p_{1}-1\right)} \mathrm{d} t
\end{aligned}
$$

for all $\varepsilon_{1} \in(0,1)$. Now we define

$$
\phi_{2}(t)=\varpi(t) \frac{y^{\prime}(t)}{y(t)}
$$

Then $\phi_{2}(t)>0$ for $t \geq t_{1}$. Using (32) and (29), we obtain

$$
\begin{aligned}
\phi_{2}^{\prime}(t)= & \frac{\varpi^{\prime}(t)}{\varpi(t)} \phi_{2}(t)+\varpi(t) \frac{y^{\prime \prime}(t)}{y(t)}-\varpi(t)\left(\frac{y^{\prime}(t)}{y(t)}\right)^{2} \\
\leq & \frac{\varpi^{\prime}(t)}{\varpi(t)} \phi_{2}(t)-\frac{1}{\varpi(t)} \phi_{2}^{2}(t) \\
& -\left(\left(1-r_{0}\right) \varepsilon_{1}\right)^{\left(p_{2}-1\right) /\left(p_{1}-1\right)} \varpi(t) y^{\left(p_{2}-1\right) /\left(p_{1}-2\right)}(t) \\
& \times \int_{t}^{\infty}\left(\frac{1}{a(t)} \int_{t}^{\infty} q(s) \frac{\sigma^{\left(p_{2}-1\right)}(s)}{s^{\left(p_{2}-1\right)}} \mathrm{d} s\right)^{1 /\left(p_{1}-1\right)} \mathrm{d} t .
\end{aligned}
$$

Thus we find

$$
\phi_{2}^{\prime}(t) \leq-\Psi_{1}(t)+\frac{\varpi^{\prime}(t)}{\varpi(t)} \vartheta(t)-\frac{1}{\varpi(t)} \phi_{2}^{2}(t) .
$$

This completes the proof. 
Theorem 3.4 Assume that $r_{0}<1$ and $\sigma(t) \leq t$. If there exist two positive functions $\varpi_{1}, \varpi \in$ $C^{1}\left(\left[t_{0}, \infty\right)\right)$ such that

$$
\int_{t_{0}}^{\infty}\left(\Psi(s)-\frac{2^{\left(p_{1}-1\right)}}{p_{1}^{p_{1}}} \frac{a(s)\left(\varpi_{1}^{\prime}(s)\right)^{p_{1}}}{\mu_{1}^{\left(p_{1}-1\right)} s^{2\left(p_{1}-1\right)} \varpi_{1}^{\left(p_{1}-1\right)}(s)}\right) \mathrm{d} s=\infty
$$

and

$$
\int_{t_{0}}^{\infty}\left(\Psi_{1}(s)-\frac{\left(\varpi^{\prime}(s)\right)^{2}}{4 \varpi(s)}\right) \mathrm{d} s=\infty
$$

then (1) is oscillatory.

Proof It is known that (9) and (18) hold in the proof of Theorem 3.3. From (9) we have that $y^{\prime \prime}$ is of one sign. From Lemma 3.1 we get that (21) holds.

Since $y^{\prime}(t)>0$, there exist $t_{2} \geq t_{1}$ and a constant $M>0$ such that

$$
y(t)>M
$$

for all $t \geq t_{2}$. From the inequality

$$
E w-F w^{(\alpha+1) / \alpha} \leq \frac{\alpha^{\alpha}}{(\alpha+1)^{\alpha+1}} E^{\alpha+1} F^{-\alpha}, \quad F>0,
$$

with $E=\varpi_{1}^{\prime}(t) / \varpi_{1}(t), F=\left(p_{1}-1\right) \mu t^{2} / 2 a^{1 /\left(p_{1}-1\right)}(t) \varpi_{1}^{1 /\left(p_{1}-1\right)}(t)$, and $x=\phi_{1}$, we get

$$
\phi_{1}^{\prime}(t) \leq-\Psi(t)+\frac{2^{\left(p_{1}-1\right)}}{p_{1}^{p_{1}}} \frac{a(t)\left(\varpi_{1}^{\prime}(t)\right)^{p_{1}}}{\mu_{1}^{\left(p_{1}-1\right)} t^{2\left(p_{1}-1\right)} \varpi_{1}^{\left(p_{1}-1\right)}(t)} .
$$

This implies that

$$
\int_{t_{1}}^{t}\left(\Psi(s)-\frac{2^{\left(p_{1}-1\right)}}{p_{1}^{p_{1}}} \frac{a(s)\left(\varpi_{1}^{\prime}(s)\right)^{p_{1}}}{\mu_{1}^{\left(p_{1}-1\right)} s^{2\left(p_{1}-1\right)} \varpi_{1}^{\left(p_{1}-1\right)}(s)}\right) \mathrm{d} s \leq \phi_{1}\left(t_{1}\right),
$$

which contradicts (30).

From Lemma 3.2 we get that (25) holds. This implies that

$$
\phi_{2}^{\prime}(t) \leq-\Psi_{1}(t)+\frac{\left(\varpi^{\prime}(t)\right)^{2}}{4 \varpi(t)} .
$$

Then we obtain

$$
\int_{t_{1}}^{t}\left(\Psi_{1}(s)-\frac{\left(\varpi^{\prime}(t)\right)^{2}}{4 \varpi(t)}\right) \mathrm{d} s \leq \phi_{2}\left(t_{1}\right)
$$

which contradicts (31). This completes the proof.

Example 3.1 Consider the equation

$$
(x(t)+(7 / 8) x(t / e))^{(4)}+q_{0} u^{-4} x\left(t / e^{2}\right)=0, \quad u \geq 1,
$$


Table 1 Conditions comparison

\begin{tabular}{lllll}
\hline Condition & $(3)$ & $(4)$ & $(5)$ & $(6)$ \\
\hline Criterion & $90>113981.3$ & $90>3561.9$ & $90>3008.5$ & $90>587.93$ \\
\hline
\end{tabular}

where $q_{0}>0$ is a constant, and

$$
p_{1}=2, \quad a(t)=1, \quad r(t)=7 / 8, \quad \delta(t)=u / e, \quad q(t)=q_{0} u^{-4}, \quad \sigma(t)=t / e^{2} .
$$

Applying conditions (3), (4), (5), and (6) to Eq. (33), we obtain Table 1.

Therefore we see that [27] enriched the results in [24-26]. Furthermore, we easily find that the conditions for oscillation in [24-26] cannot be applied to (35) and (36). Therefore our results are new.

Example 3.2 Consider the differential equation

$$
\left(\left(\left(x+r_{0} x(\varpi t)\right)^{\prime \prime \prime}\right)^{\left(p_{1}-1\right)}\right)^{\prime}+\frac{q_{0}}{t^{3 p_{1}-2}} x(\lambda t)=0, \quad t \geq 1
$$

where $\varpi, \lambda \in(0,1]$ and $r_{0}, q_{0}>0$. Let $a(t)=1, r(t)=r_{0}, \delta(t)=\varpi t, \sigma(t)=\lambda t$, and $q(t)=$ $q_{0} / t^{3 p_{1}-2}$. We easily see that

$$
\widehat{q}(t)=q_{0} \lambda^{3 p_{1}-2} \frac{1}{t^{3 p_{1}-2}}
$$

By Corollary 3.1 equation (34) is oscillatory if

$$
q_{0} \ln \frac{1}{\lambda}>\left(p_{1}-1\right)\left(\frac{\varpi+r_{0}^{\left(p_{1}-1\right)}}{\varpi}\right) \frac{6^{\left(p_{1}-1\right)}}{\lambda^{3\left(p_{1}-1\right)} \mathrm{e}} .
$$

By Corollary 3.2, if

$$
q_{0} \ln \frac{1}{\lambda}>\frac{1}{\left(1-r_{0}\right)^{\left(p_{1}-1\right)}} \frac{6^{\left(p_{1}-1\right)}}{\lambda^{3\left(p_{1}-1\right)} \mathrm{e}}
$$

then (34) is oscillatory.

Finally, setting $\varpi_{1}(s):=t^{3\left(p_{1}-1\right)}$ and $\varpi(t):=t^{2}$, we have

$$
\Psi(t)=q_{0}\left(1-r_{0}\right)^{\left(p_{1}-1\right)} \lambda^{3\left(p_{1}-1\right)} \frac{1}{s}
$$

and

$$
\Psi_{1}(t):=\frac{1}{2}\left(\frac{q_{0}}{3\left(p_{1}-1\right)}\right)^{1 /\left(p_{1}-1\right)}\left(1-r_{0}\right) \lambda
$$

By Theorem 3.4 equation (34) is oscillatory if

$$
q_{0}\left(1-r_{0}\right)^{\left(p_{1}-1\right)} \lambda^{3\left(p_{1}-1\right)}>2^{\left(p_{1}-1\right)} 3^{p_{1}}\left(\frac{\left(p_{1}-1\right)}{p_{1}}\right)^{p_{1}}
$$


and

$$
q_{0}>\left(\frac{2}{\left(1-r_{0}\right) \lambda}\right)^{\left(p_{1}-1\right)} 3\left(p_{1}-1\right)
$$

\section{Conclusion}

In this paper, we consider the oscillation and asymptotic behavior of a class of fourthorder Emden-Fowler neutral differential equations. Using the Riccati transformation and comparison method, we establish new oscillation conditions for the solutions of fourthorder neutral differential equations. Our results unify and extend some known results for differential equations. In the future work, we will discuss the oscillatory behavior of these equations by using comparing technique with second-order equations.

\section{Acknowledgements}

The authors thank the editors and the reviewers for their useful comments.

Funding

The authors received no direct funding for this work.

Availability of data and materials

Not applicable.

Competing interests

The authors declare that they have no competing interests.

Authors' contributions

The authors declare that they read and approved the final manuscript.

\section{Authors' information}

Not applicable.

\section{Author details}

${ }^{1}$ Department of Mathematics, Faculty of Science, Hadhramout University, Hadhramout 50512, Yemen. ${ }^{2}$ Department of Mathematics, Faculty of Education, Seiyun University, Hadhramout 50512, Yemen. ${ }^{3}$ Department of Mathematics, Faculty of Science, University of Hafr Al Batin, P.O. Box 1803, Hafar Al Batin 31991, Saudi Arabia.

\section{Publisher's Note}

Springer Nature remains neutral with regard to jurisdictional claims in published maps and institutional affiliations.

Received: 22 December 2020 Accepted: 11 February 2021 Published online: 25 February 2021

\section{References}

1. Hale, J.K.: Theory of Functional Differential Equations. Springer, New York (1977)

2. Bazighifan, O., Kumam, P.: Oscillation theorems for advanced differential equations with $p$-Laplacian like operators. Mathematics 8, 821 (2020)

3. Bazighifan, O., Abdeljawad, T.: Improved approach for studying oscillatory properties of fourth-order advanced differential equations with $p$-Laplacian like operator. Mathematics 8, 1-11 (2020)

4. Agarwal, R.P., Bohner, M., Li, T., Zhang, C.: Oscillation of second-order Emden-Fowler neutral delay differential equations. Ann. Mat. Pura Appl. (4) 193(6), 1861-1875 (2014)

5. Bohner, M., Hassan, T.S., Li, T.: Fite-Hille-Wintner-type oscillation criteria for second-order half-linear dynamic equations with deviating arguments. Indag. Math. 29(2), 548-560 (2018)

6. Dzurina, J., Grace, S.R., Jadlovska, I., Li, T.: Oscillation criteria for second-order Emden-Fowler delay differential equations with a sublinear neutral term. Math. Nachr. 293(5), 910-922 (2020)

7. Li, T., Pintus, N., Viglialoro, G.: Properties of solutions to porous medium problems with different sources and boundary conditions. Z. Angew. Math. Phys. 70(3), 86, 1-18 (2019)

8. Li, T., Rogovchenko, Yu.V.: Oscillation of second-order neutral differential equations. Math. Nachr. 288(10), 1150-1162 (2015)

9. Li, T., Rogovchenko, Yu.V.: Oscillation criteria for second-order superlinear Emden-Fowler neutral differential equations. Monatshefte Math. 184(3), 489-500 (2017)

10. Zhang, C., Agarwal, R.P., Li, T.: Oscillation and asymptotic behavior of higher-order delay differential equations with p-Laplacian like operators. J. Math. Anal. Appl. 409(2), 1093-1106 (2014)

11. Agarwal, R.P., Bohner, M., Li, T., Zhang, C.: A new approach in the study of oscillatory behavior of even-order neutral delay differential equations. Appl. Math. Comput. 225, 787-794 (2013) 
12. Li, T., Rogovchenko, Yu.V.: Oscillation criteria for even-order neutral differential equations. Appl. Math. Lett. 61, 35-41 (2016)

13. Chatzarakis, G.E., Jadlovska, I., Li, T.: Oscillations of differential equations with non-monotone deviating arguments. Adv. Differ. Equ. 2019, 233, 1-20 (2019)

14. Chatzarakis, G.E., Li, T.: Oscillation criteria for delay and advanced differential equations with nonmonotone arguments. Complexity 2018, Article ID 8237634 (2018)

15. Li, T., Rogovchenko, Yu.V.: On asymptotic behavior of solutions to higher-order sublinear Emden-Fowler delay differential equations. Appl. Math. Lett. 67, 53-59 (2017)

16. Zhang, C., Li, T., Sun, B., Thandapani, E.: On the oscillation of higher-order half-linear delay differential equations. Appl. Math. Lett. 24(9), 1618-1621 (2011)

17. Li, T., Rogovchenko, Yu.V.: On the asymptotic behavior of solutions to a class of third-order nonlinear neutral differential equations. Appl. Math. Lett. 105, 106293 1-7 (2020)

18. Chatzarakis, G.E.: Oscillation test for linear deviating differential equations. Appl. Math. Lett. 98, 352-358 (2019)

19. Chatzarakis, G.E., Dzurina, J., Jadlovska, I.: New oscillation criteria for second-order half-linear advanced differential equations. Appl. Math. Comput. 347, 404-416 (2019)

20. Chatzarakis, G.E., Dzurina, J., Jadlovska, I.: A remark on oscillatory results for neutral differential equations. Appl. Math. Lett. 90, 124-130 (2019)

21. Zhang, C., Agarwal, R.P., Bohner, M., Li, T.: Oscillation of fourth-order delay dynamic equations. Sci. China Math. 58(1), $143-160$ (2015)

22. Bazighifan, O., Alotaibi, H., Mousa, A.A.A.: Neutral delay differential equations: oscillation conditions for the solutions. Symmetry 13, $101(2021)$

23. Bazifghifan, O., Ramos, $\mathrm{H}$.: On the asymptotic and oscillatory behavior of the solutions of a class of higher-order differential equations with middle term. Appl. Math. Lett. 107, 106431 (2020)

24. Zafer, A.: Oscillation criteria for even order neutral differential equations. Appl. Math. Lett. 11, 21-25 (1998)

25. Zhang, Q., Yan, J.: Oscillation behavior of even order neutral differential equations with variable coefficients. Appl. Math. Lett. 19, 1202-1206 (2006)

26. Xing, G., Li, T., Zhang, C.: Oscillation of higher-order quasi linear neutral differential equations. Adv. Differ. Equ. 2011, 45, 1-10 (2011)

27. Moaaz, O., Awrejcewicz, J., Bazighifan, O.: A new approach in the study of oscillation criteria of even-order neutral differential equations. Mathematics 8, 197 (2020)

28. Li, T., Baculikova, B., Dzurina, J., Zhang, C.: Oscillation of fourth order neutral differential equations with $p$-Laplacian like operators. Bound. Value Probl. 56, 41-58 (2014)

29. Bazighifan, O.: On the oscillation of certain fourth-order differential equations with $p$-Laplacian like operator. Appl. Math. Comput. 386, 125475 (2020)

30. Agarwal, R.P., Bazighifan, O., Ragusa, M.A.: Nonlinear neutral delay differential equations of fourth-order: oscillation of solutions. Entropy 23, 129 (2021)

31. Liu, S., Zhang, Q., Yu, Y.: Oscillation of even-order half-linear functional differential equations with damping. Comput Math. Appl. 61, 2191-2196 (2011)

32. Agarwal, R., Grace, S., O'Regan, D.: Oscillation criteria for certain $n$th order differential equations with deviating arguments. J. Math. Anal. Appl. 262,601-622 (2001)

33. Bazighifan, O.: Oscillatory applications of some fourth-order differential equations. Math. Methods Appl. Sci. 43(17), 10276-10286 (2020). https://doi.org/10.1002/mma.6694

34. Bazighifan, O., Chatzarakis, G.E.: Oscillatory and asymptotic behavior of advanced differential equations. Adv. Differ. Equ. 2020, 414 (2020)

35. Chatzarakis, G.E., Elabbasy, E.M., Bazighifan, O.: An oscillation criterion in 4th-order neutral differential equations with a continuously distributed delay. Adv. Differ. Equ. 336, 1-9 (2019)

36. Moaaz, O., Elabbasy, E.M., Muhib, A.: Oscillation criteria for even-order neutral differential equations with distributed deviating arguments. Adv. Differ. Equ. 2019, 297 (2019)

37. Philos, Ch.G.: On the existence of non-oscillatory solutions tending to zero at $\infty$ for differential equations with positive delays. Arch. Math. 36, 168-178 (1981)

38. Zhang, C., Agarwal, R.P., Bohner, M., Li, T.: New results for oscillatory behavior of even-order half-linear delay differential equations. Appl. Math. Lett. 26, 179-183 (2013)

39. Ladde, G.S., Lakshmikantham, V., Zhang, B.G.: Oscillation Theory of Differential Equations with Deviating Arguments. Dekker, New York (1987)

40. Chatzarakis, G.E., Grace, S.R., Jadlovska, I., Li, T., Tunc, E.: Oscillation criteria for third-order Emden-Fowler differential equations with unbounded neutral coefficients. Complexity 2019, Article ID 5691758 (2019)

41. Agarwal, R., Grace, S., O'Regan, D.: Oscillation Theory for Difference and Functional Differential Equations. Kluwer Academic, Dordrecht (2000)

42. Baculikova, B., Dzurina, J.: Oscillation theorems for second-order nonlinear neutral differential equations. Comput. Math. Appl. 62, 4472-4478 (2011) 